\title{
Esophageal Hematoma Associated with the Bolus Ingestion of Hot Coffee
}

\author{
Yorinari Ochiai, Daisuke Kikuchi and Shu Hoteya
}

\begin{abstract}
:
A 59-year-old woman presented with pharyngeal discomfort and dysphagia starting the previous day. Esophagogastroduodenoscopy revealed a longitudinal reddish area and hematoma mainly on the left wall of the esophagus. On the previous day, she had felt a piece of meat sticking in her throat while eating; she therefore rapidly gulped down some hot coffee to hasten the passage of the meat. Based on the history, we diagnosed her endoscopic findings as esophageal hematoma and thermal injury associated with hot coffee. We herein describe a case of an acute esophageal hematoma and thermal injury and the clinical course following endoscopy.
\end{abstract}

Key words: esophageal hematoma, thermal injury, hot coffee

(Intern Med 59: 2701-2704, 2020)

(DOI: 10.2169/internalmedicine.5110-20)

\section{Introduction}

Esophageal hematoma and thermal injury are both rare benign esophageal diseases. Esophageal hematoma is associated with multiple causes, including hurried swallowing of an overly compact or bulky bolus (1). In contrast, there are some reports of esophageal thermal injury caused by hot liquids or solid foods, in which endoscopic findings show exfoliative esophagitis (2) or alternating pink and white bands with a candy-cane appearance (3). However, esophageal hematoma caused by hot liquid is not common. In addition, the clinical course of this injury remains unclear. We observed a rare case of esophageal longitudinal hematoma associated with the bolus ingestion of hot coffee. We herein present the clinical course and serial endoscopic findings of the patient.

\section{Case Report}

A 59-year-old woman presented to our hospital with pharyngeal discomfort and dysphagia beginning the previous day. She had no past medical history and was not taking any regular medication. One day prior to her visit, she had felt a piece of meat sticking in her throat while eating. She there- fore drank very hot coffee (about 70-80 mL, immediately after heating) in a single gulp in an attempt to hasten the passage of the meat. On presentation, her vital signs were within the normal limits and laboratory blood tests were unremarkable. We performed esophagogastroduodenoscopy (EGD) two days after her visit, revealing a longitudinal hematoma throughout the esophagus (Fig. 1). Apart from the hematoma, there were areas with alternating linear pink and white bands, referred to as candy-cane appearance in the other areas of the esophagus (Fig. 1, the right part of A and B). Computed tomography revealed extensive longitudinal swelling of the left wall throughout the esophagus. This was considered to be esophageal hematoma (Fig. 2). We made a diagnosis of esophageal hematoma caused by thermal injury from hot liquid. We treated her with a proton pump inhibitor (PPI) to prevent acid reflux and sucralfate hydrate for mucosal protection, while prohibiting all oral intake. Her symptoms gradually improved and disappeared after a week from first presentation. A second EGD was performed on that day, and it revealed an esophageal ulcer where the hematoma had been seen at the previous EGD (Fig. 3). She was discharged one week after the second EGD when she was able to eat soft meals. After discharge, she continued the PPI and sucralfate hydrate without any symptoms. A third EGD 6 weeks from first presentation showed a longitudinal 

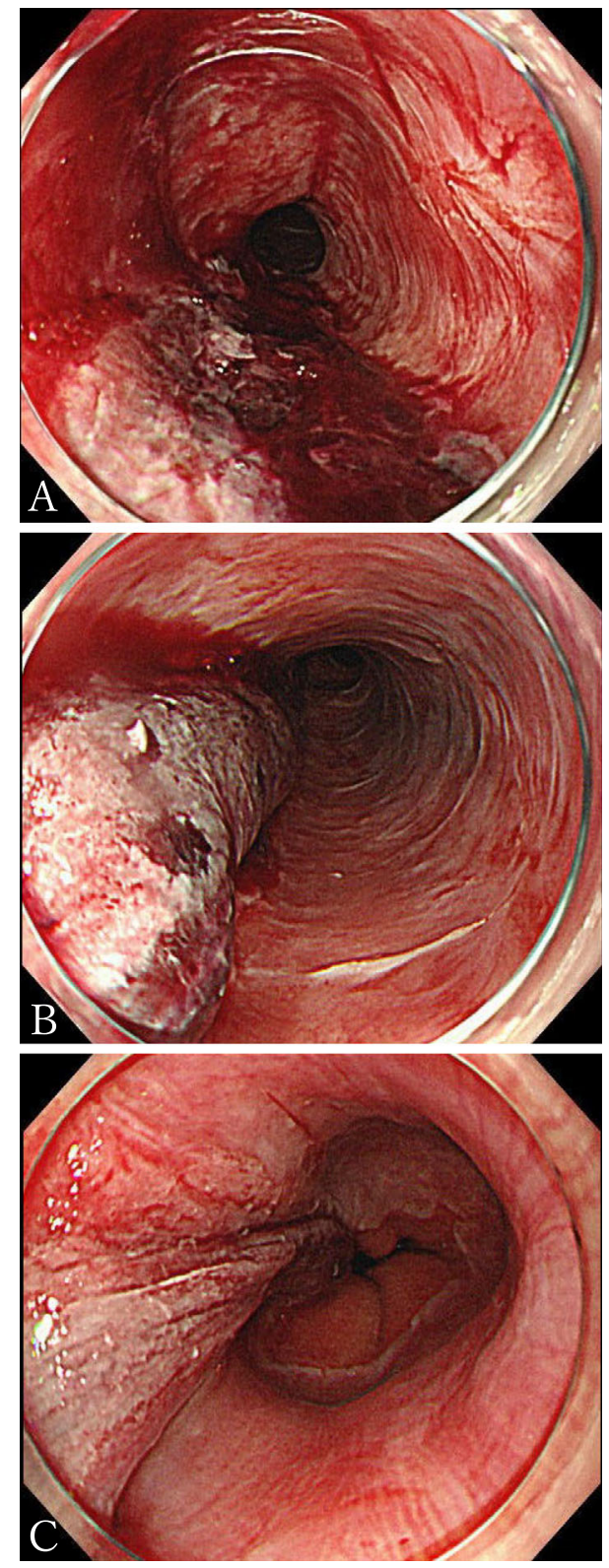

Figure 1. Esophageal hematoma at the first endoscopy (3 days after event). (A) Upper part of the esophagus, (B) Middle part of the esophagus, $(C)$ Lower part of the esophagus. ulcer scar in the esophagus without any stricture (Fig. 4).

\section{Discussion}

Esophageal hematoma was first reported by Williams (4). It is said that the cause of esophageal hematoma can be divided into five categories: related to abnormal hemostasis, emetogenic, traumatic/iatrogenic, related to aortic disease, or spontaneous (1). Vomiting, coughing, sneezing, hurried swallowing of an overly compact or bulky bolus, or lifting a heavy weight are considered to be precipitating factors. In contrast, thermal injury of the esophagus can be caused by hot liquids or hot solid food (5), and endoscopic findings show thick, whitish pseudomembranous mucosa with intervening hyperemic mucosa (6) and a candy-cane appearance (3).

Our patient showed endoscopic findings of esophageal hematoma and esophageal thermal injury. It could be argued that both swallowing meat and drinking hot coffee were causes because the sudden change in esophageal pressure by swallowing meat was one of the risks. However, the esophageal hematoma and other findings of thermal injury were worse in the upper part of the esophagus, while the site of injury of the esophagus is supposed to be mainly distal when the etiology is considered to be a sudden change in intrathoracic and esophageal pressure such as with bulky bolus ingestion (1). Based on these findings, we speculated that the main cause of the longitudinal esophageal hematoma in this case was the bolus ingestion of hot coffee. Esophageal hematoma caused by thermal injury is rare, and we could not identify any previous reports in the international literature, although we found another case from Japan (7). The previous case also presented with a longitudinal hematoma throughout the esophagus. Previous literature reported that the site of injury might be related to adjacent structures, such as the trachea and heart (8). Esophageal hematoma of the left wall in our patient might be due to the surrounding organs, such as the trachea and vertebra. The cause of hematoma due to thermal injury is considered to be related to mechanical stimulation; however, this remains un-
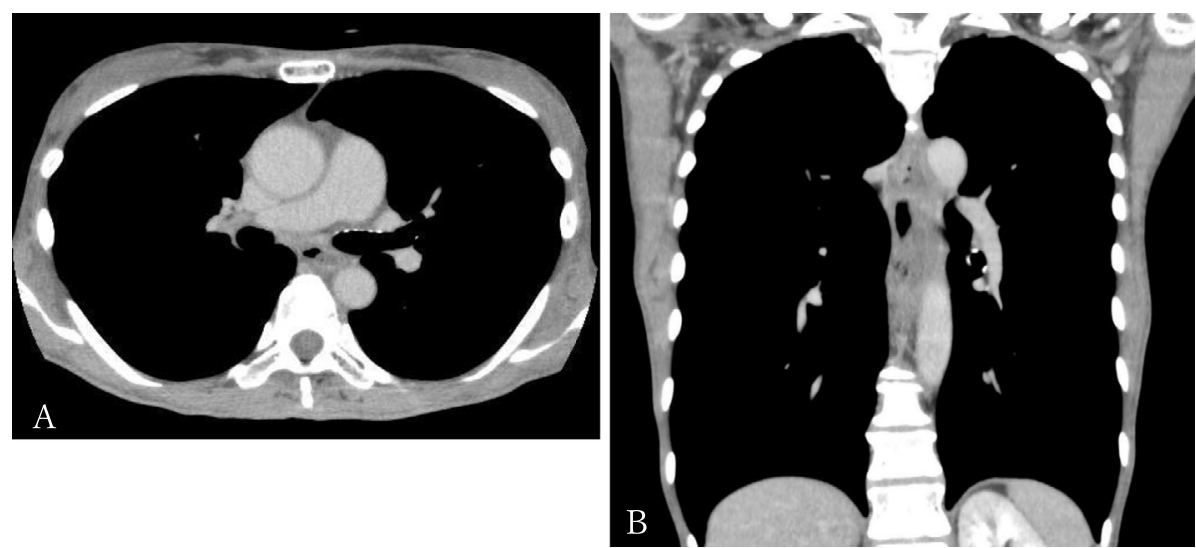

Figure 2. (A) Axial, (B) coronal computed tomography scans after the first endoscopy. 


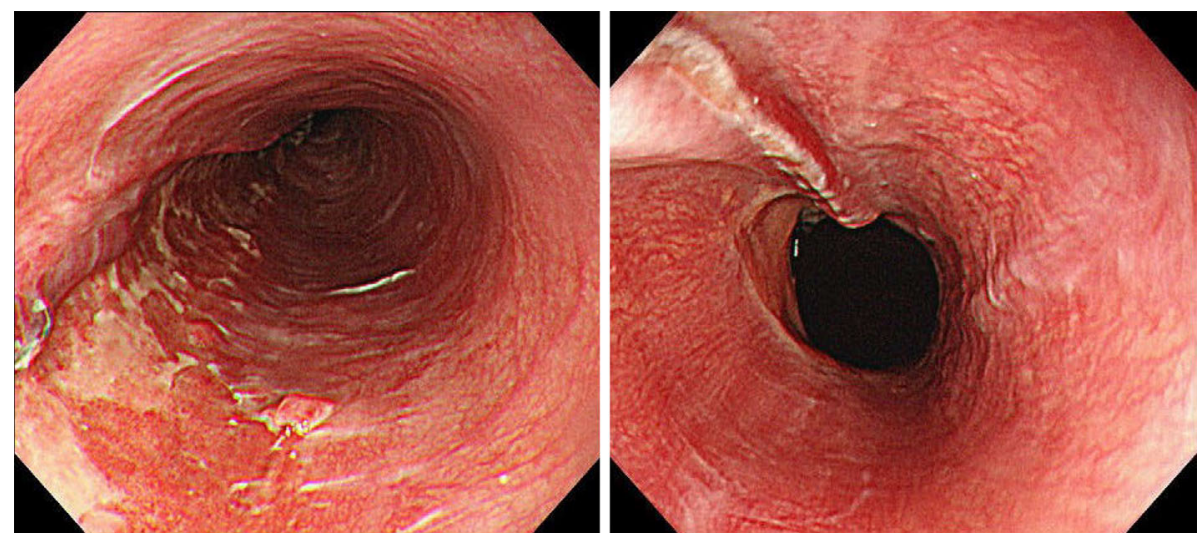

Figure 3. Esophageal ulcer at second endoscopy (1 week after event).

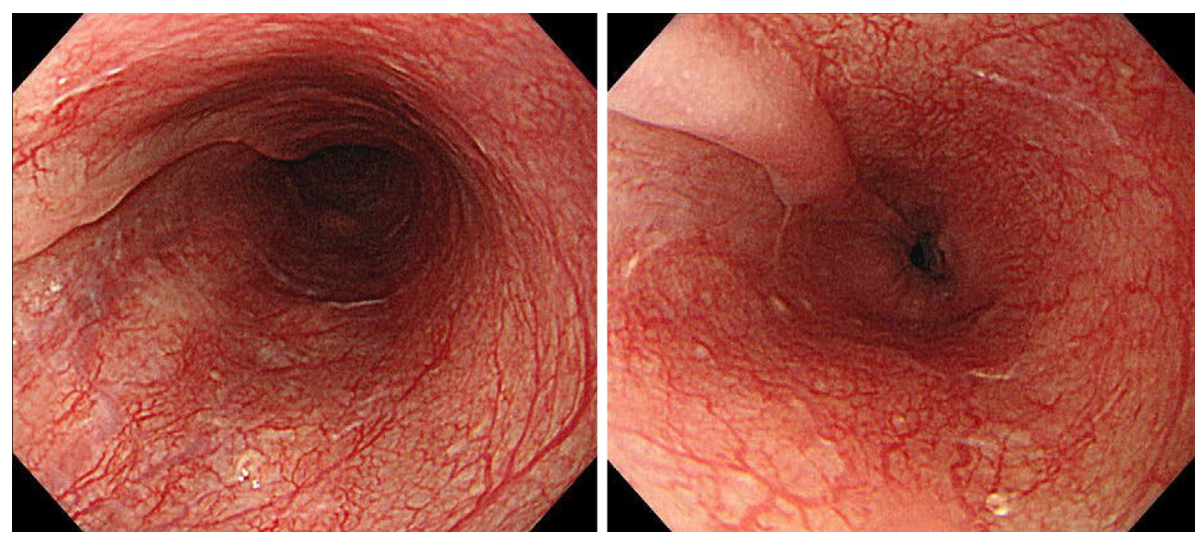

Figure 4. Esophageal scar at third endoscopy (6 weeks after event).

clear.

The treatment for esophageal hematoma caused by thermal injury is conservative treatment with fasting and medications for mucosal protection, such as PPIs, which were used in our case and the other reported case. The prognoses of these two patients were very good without any stricture of the esophagus. However, a case of severe esophageal stricture due to thermal injury treated with esophagectomy was reported (9). This patient had also gulped a cup of hot coffee, and this event created a pin-hole like stricture located $19 \mathrm{~cm}$ distally from the incisors, leading to an eventual esophagectomy six months later. On histopathological examination, marked fibrosis extending to the muscularis propria and adventitia was observed all along the esophagus. By contrast, in the previous report, histologic studies of thermal injury of the esophagus with a candy-cane appearance showed that the mucosa was characterized by a superficial layer of necrotic and nonviable epithelium adherent to the underlying viable squamous epithelial cells (3). Our patient had an esophageal hematoma located only on the left wall of the esophagus, which resulted in an ulcer extending approximately 2/12th of the circumference. Therefore, we speculated that significant stricture was not observed in our patient. The presence of a wide circumferential extension of ulceration or hematoma may be speculated to be associated with severe stricture within a few months. Such findings must be identified during first endoscopy and follow-up endoscopy must be performed frequently until confirmation of wound healing. However, the difference in esophageal strictures between our patient and the previous one is still unclear. The differences might be due to the temperature, amount, type of beverages, and drinking speed because the previous patient who underwent esophagectomy had consumed more than twice as much hot coffee as our patient in a single gulp. Additional accumulation and the analysis of similar cases is therefore required.

In conclusion, we herein described a rare case of esophageal hematoma associated with thermal injury due to bolus ingestion of hot coffee, which was successfully managed by conservative treatment.

The authors state that they have no Conflict of Interest (COI).

\section{References}

1. Cao DT, Reny JL, Lanthier N, Frossard JL. Intramural hematoma of the esophagus. Case Rep Gastroenterol 6: 510-517, 2012.

2. Schertl F, Lewerenz B, Schepp W. Exfoliative esophagitis caused by habitual ingestion of very hot coffee. Clin Gastroenterol Hepatol 16: e119, 2018.

3. Dutta SK, Chung KY, Bhagavan BS. Thermal injury of the esophagus. N Engl J Med 339: 480-481, 1998.

4. Williams B. Case report; oesophageal laceration following remote 
trauma. Bri J Radiol 30: 666-668, 1957.

5. Chung WC, Paik CN, Jung JH, Kim JD, Lee KM, Yang JM. Acute thermal injury of the esophagus from solid food: clinical course and endoscopic findings. J Korean Med Sci 25: 489-491, 2010.

6. Choi EK, Lee GH, Jung HY, et al. The healing course of thermal esophageal injury: a case report. Gastrointesti Endosc 62: 158-160, 2005.

7. Baba T, Shida A, Aoki H, Yokota T, Kurihara H, Suzuki H. A case of an elderly man on low-dose aspirin therapy who developed an esophageal intramural hematoma due to a thermal burn. Gastroenterol Endosc 5: 2213-2218, 2012 (in Japanese).
8. Cullen SN, McIntyre AS. Dissecting intramural haematoma of the oesophagus. Eur J Gastroenterol Hepatol 12: 1151-1162, 2000.

9. Kitajima T, Momose K, Lee S, et al. Benign esophageal stricture after thermal injury treated with esophagectomy and ileocolon interposition. World J Gastroenterol 20: 9205-9209, 2014.

The Internal Medicine is an Open Access journal distributed under the Creative Commons Attribution-NonCommercial-NoDerivatives 4.0 International License. To view the details of this license, please visit (https://creativecommons.org/licenses/ by-nc-nd/4.0/).

(C) 2020 The Japanese Society of Internal Medicine Intern Med 59: 2701-2704, 2020 